\title{
In-Hospital Postoperative Pneumonia Following Geriatric Intertrochanteric Fracture Surgery: Incidence and Risk Factors
}

This article was published in the following Dove Press journal:

Clinical Interventions in Aging

\author{
Kuo Zhao ${ }^{1-3}$ \\ Junzhe Zhang ${ }^{1-3}$ \\ Junyong $\mathrm{Li}^{1-3}$ \\ Jialiang Guo ${ }^{1-3}$ \\ Hongyu Meng ${ }^{1-3}$ \\ Yanbin Zhu ${ }^{\mathrm{I}-3}$ \\ Yingze Zhang ${ }^{\text {I-3 }}$ \\ Zhiyong Hou ${ }^{1-3}$
}

'Department of Orthopaedic Surgery, Third Hospital of Hebei Medical University, Shijiazhuang 05005I, Hebei,

People's Republic of China; ${ }^{2} \mathrm{Key}$ Laboratory of Biomechanics of Hebei Province, Shijiazhuang 05005I, Hebei, People's Republic of China; ${ }^{3}$ Orthopaedic Research Institution of Hebei Province, Shijiazhuang 05005I, Hebei, People's Republic of China
Correspondence: Yingze Zhang; Zhiyong Hou

Email suryzz@|26.com; surzyh@I26. com
Purpose: The in-hospital death rate in cases of hip fracture ranges from $6 \%$ to $10 \%$. Pneumonia is a serious complication for hip fracture patients that contributes to longer hospital stays and higher mortality rates; however, the prevalence and risk factors are not well established. To address this issue, the present study investigated the incidence of and risk factors for in-hospital postoperative pneumonia (IHPOP) following geriatric intertrochanteric fracture surgery.

Patients and Methods: Information on 1495 geriatric patients ( $>65$ years) who underwent intertrochanteric fracture surgery at our hospital between October 2014 and December 2018 was extracted from a prospective hip fracture database and reviewed. Demographic information, clinical variables including surgical data, and preoperative laboratory indices that could potentially influence IHPOP were analyzed. Receiver operating characteristic curve analysis was performed and the optimum cutoff value for quantitative data was determined. Univariate and multivariate analyses were carried out to identify risk factors for IHPOP.

Results: The incidence of IHPOP following geriatric intertrochanteric fracture surgery was 3.5\% (53/1495 cases). The multivariate analysis showed that age $>82$ years (odds ratio $[\mathrm{OR}]=2.54$, $\mathrm{p}=0.004)$, male sex $(\mathrm{OR}=2.13, \mathrm{p}=0.017)$, chronic respiratory disease $(\mathrm{OR}=5.02, \mathrm{p}<0.001)$, liver disease $(\mathrm{OR}=3.39, \mathrm{p}=0.037)$, urinary tract infection $(\mathrm{OR}=8.46, \mathrm{p}=0.005)$, creatine kinase $(\mathrm{CK})$ $\mathrm{MB}>20 \mathrm{U} / 1(\mathrm{OR}=2.31, \mathrm{p}=0.020), \mathrm{B}$-type natriuretic peptide $(\mathrm{BNP}) \geq 75 \mathrm{ng} / \mathrm{l}(\mathrm{OR}=4.02, \mathrm{p}=0.001)$, and $\mathrm{D}$-dimer $>2.26 \mathrm{mg} / \mathrm{l}(\mathrm{OR}=2.69, \mathrm{p}=0.002)$ were independent risks factor for the incidence of IHPOP following geriatric intertrochanteric fracture surgery.

Conclusion: The incidence of IHPOP was 3.5\% following geriatric intertrochanteric fracture surgery; age, male sex, chronic respiratory disease, liver disease, urinary tract infection, CKMB, BNP, and D-dimer were significant risk factors. Targeted preoperative management based on these factors could reduce the risk of IHPOP and mortality in these patients.

Keywords: intertrochanteric fracture, postoperative pneumonia, in-hospital complication, geriatric population, risk factor

\section{Introduction}

Intertrochanteric fracture is a common type of osteoporotic hip fracture in the geriatric population, accounting for approximately $50 \%$ of hip fractures and $3.13 \%$ of all fractures in adults. ${ }^{1,2}$ With the aging of the global population, the incidence of intertrochanteric fracture is increasing and its treatment consumes a growing proportion of healthcare expenditure. ${ }^{3}$ While intertrochanteric fracture surgery can be successfully performed under most conditions with an intramedullary or extramedullary device, ${ }^{4}$ the 
30 -day and 1-year mortality is $7.7 \%$ and $26 \%$, respectively; ${ }^{5}$ preexisting comorbidities at the time of fracture and postoperative complications are the major causes of these high rates. ${ }^{6}$ One study found that postoperative infection was the leading cause of death in hip fracture patients. ${ }^{7}$ Pneumonia is a devastating infection in elderly patients and is among the most frequent postoperative complications following geriatric intertrochanteric fracture surgery. ${ }^{8,9}$ It was reported that the rate of pneumonia within 30 days after hip fracture surgery ranges from $4.1 \%$ to $11.9 \%{ }^{10,11}$ Previous studies have shown that postoperative pneumonia (POP) is associated with increased 30-day and 1-year mortality; ${ }^{6,7,12}$ the 30-day mortality for hip fracture patients who developed POP is $27-43 \%{ }^{10,13}$ which is 4.18 times higher than in patients without POP. ${ }^{12}$

Factors contributing to increased risk of POP following hip fracture surgery have been investigated in previous studies. ${ }^{7,10,13}$ However, these studies described the surgical treatment of both intertrochanteric and femoral neck fractures and there were significant differences in the demographic profile of the study population, fixation device that was used, and rehabilitation program. ${ }^{1,5,14}$ The fixation device had a significant influence on the incidence of POP after hip fracture, ${ }^{10}$ resulting in distinct risk factors for POP between the 2 fracture types. Meanwhile, few studies have examined the incidence and risk factors of POP following geriatric intertrochanteric fracture surgery. One reported an association between C-reactive protein (CRP) and postoperative systemic infections in cases of intertrochanteric fracture but did not separately evaluate the relationship between CRP and POP. ${ }^{15}$ Intramedullary fixation was shown to increase the rate of POP, but there were differences in complication rates between patients with intertrochanteric femoral fracture treated with an intramedullary vs extramedullary device. ${ }^{16}$ Potential risk factors for POP in patients with hip fractures are sex, advanced age, body mass index (BMI), smoking history, preexisting pulmonary disease, cerebrovascular disease, diabetes, chronic kidney disease, hypoproteinemia, anemia, number of comorbidities, American Society of Anesthesiologists (ASA) score $\geq 3$, mechanical ventilation, brain injury, previous infection, time from admission to surgery, fixation method, and certain laboratory indices. ${ }^{10,11,15,17-20}$

Most intertrochanteric fracture patients are of advanced age, fragile, and have a high risk of death. Internal factors and surgical trauma contribute to the generally poor outcome of patients who develop POP following generic intertrochanteric fracture. The purpose of this study was to detect the incidence of in-hospital postoperative pneumonia (IHPOP) following geriatric intertrochanteric fracture surgery. Secondarily, we aimed to detect whether advanced age and implant (intramedullary devices or extramedullary devices) were independent risk factors for the development of IHPOP, which may contribute to take individual treatment to prevent the developing of IHPOP in geriatric intertrochanteric fracture patients.

\section{Patients and Methods}

\section{Patients}

Information on geriatric patients ( $\geq 65$ years) who underwent surgery for intertrochanteric fracture at our hospital (a level I trauma teaching hospital) between October 2014 and December 2018 were extracted from a prospective database. A total of 1558 cases were reviewed, and 1495 were analyzed (Figure 1). The exclusion criteria were as follows: (1) received conservative treatment; (2) had preoperative pneumonia; (3) had periprosthetic fractures; (4) had old fractures (time from injury to surgery $>21$ days); (5) had multiple fractures; and (6) incomplete data. The patients in our study were divided into 2 groups: those who developed IHPOP and those without IHPOP. The study was approved by the institutional ethics committee of the Third Hospital of Hebei Medical University, and all procedures were performed in accordance with the principles outlined in the Helsinki Declaration. Informed consent was obtained from all patients.

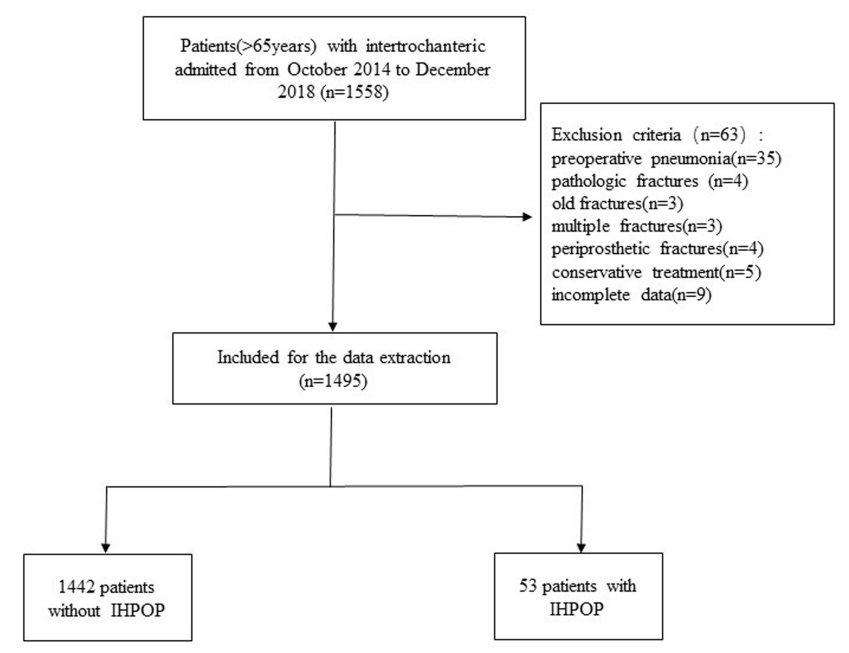

Figure I The flow chart for the selection of study participants. 


\section{Methods}

IHPOP was defined as pneumonia occurring during hospitalization following intertrochanteric fracture surgery in the absence of preoperative pneumonia. At least 1 of the following criteria was required for diagnosis: (1) new or progressive respiratory symptoms such as coughing, expectoration, and shortness of breath; (2) fever or hypothermia $\left(>38^{\circ} \mathrm{C}\right.$ or $<36^{\circ}$ C); (3) new infiltrations in postoperative chest X-rays or by computed tomography; (4) new signs of lung consolidation or moist rale detected by physical examination after surgery; (5) positive sputum or blood sample; (6) leukocytosis or leukopenia $\left(>10 \times 10^{9} / 1\right.$ or $\left.<4 \times 10^{9} / 1\right) .{ }^{10,11,13,17}$

More than 90 variables that could affect the incidence of POP were analyzed in our study, including demographic and fracture characteristics, preoperative blood biochemical indices, and surgical data. Demographic characteristics included age, sex, BMI, residential location (rural or urban), medical complications, smoking history, and disease (eg, cancer). The medical comorbidities were hypertension, circulatory abnormalities (coronary heart disease and arrhythmia), cerebrovascular disease (hemorrhagic and ischemic encephalopathy), diabetes mellitus (type 1 and type 2 diabetes), respiratory disorders (chronic bronchitis, chronic obstructive pulmonary disease and bronchiectasis), liver diseases (viral hepatitis and cirrhosis), kidney diseases (glomerulonephritis and chronic renal failure), rheumatologic disease. The number of comorbidities (hypertension; circulatory abnormalities; type 2 diabetes; respiratory disorders; and cerebrovascular, liver, kidney, and rheumatologic diseases) was recorded as 0,1 , or $\geq 2$. The ASA score of each patient was also obtained. Fracture characteristics and surgical variables were reviewed including time from admission to surgery, duration of surgery, type of anesthesia, implant type (intramedullary or extramedullary device), intraoperative blood loss, etc. Preoperative laboratory indices were measured at the time of admission. A serum albumin level $<35 \mathrm{~g} / \mathrm{L}$ was defined as malnutrition. ${ }^{21}$

\section{Statistical Analysis}

Receiver operating characteristic (ROC) curve analysis was used to determine the optimum cut-off value for quantitative data including age, BMI, and time to surgery before subsequent analysis. We then applied descriptive statistics to the entire cohort. Continuous variables conforming to a normal distribution are presented as mean \pm standard deviation and were analyzed with the Student's $t$-test or Mann-Whitney $U$-test; non-normally distributed

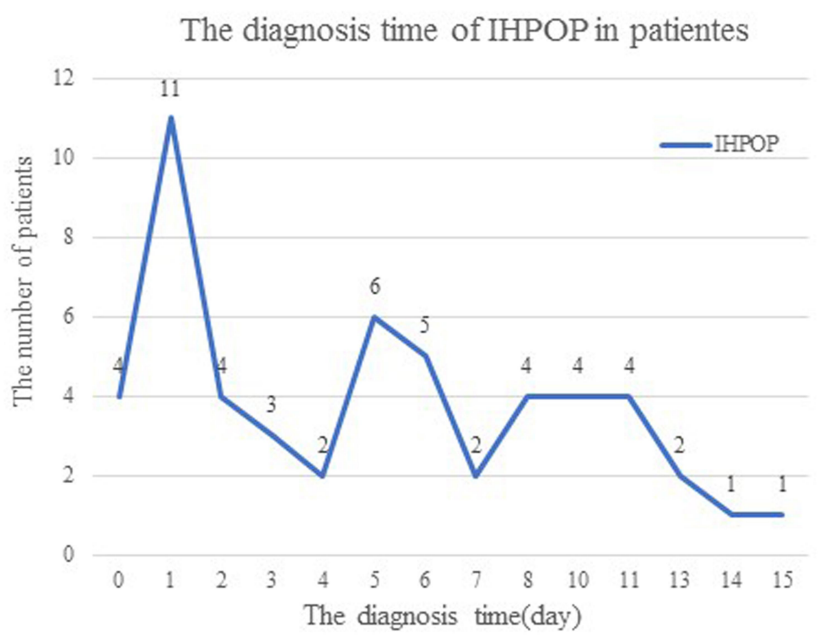

Figure 2 The diagnosis time of IHPOP in patients.

variables are presented as median values with quartiles; and categorical variables are presented as frequency and percentage and were analyzed with the chi-squared test or Fisher's exact test. After univariate analysis, potential risk factors were included in a multivariate logistic regression model to identify independent predictors of IHPOP. All statistical analyses were performed with SPSS v23.0 software (IBM, Armonk, NY, USA). A p value $<0.05$ was considered significant.

\section{Results}

\section{Clinical Parameters}

A total of 1495 cases of intertrochanteric fracture were analyzed in this study. The incidence of IHPOP following geriatric intertrochanteric fracture surgery was 3.5\% (53/ 1495 cases), while that of preoperative POP was $2.3 \%$ (35/ 1530 patients). The earliest diagnosis of IHPOP was on the day of surgery, and the latest was 15 days after surgery. The highest number of IHPOP cases (11 patients, 20.8\%) was recorded on the first day after operation. The time to

Table I Optimum Cutoff Value of Continuous Variables Detected by the ROC Analysis

\begin{tabular}{|l|l|l|l|l|}
\hline Variables & $\begin{array}{l}\text { Cutoff } \\
\text { Value }\end{array}$ & $\begin{array}{l}\text { Area Under the } \\
\text { ROC Curve } \\
\text { (AUC) }\end{array}$ & P value & $\mathbf{9 5 \% ~ C l ~}$ \\
\hline Age (years) & 82 & 0.673 & 0.000 & $0.588-0.758$ \\
BNP (ng/L) & 75 & 0.676 & 0.000 & $0.602-0.750$ \\
LYM (\%) & 5.2 & 0.383 & 0.013 & $0.302-0.465$ \\
D-Dimer(mg/L) & 2.26 & 0.626 & 0.000 & $0.537-0.714$ \\
\hline
\end{tabular}

Abbreviations: $\mathrm{ROC}$, receiver-operating characteristic; $\mathrm{Cl}$, confidence interval; BNP, brain natriuretic peptide; LYM, lymphocyte. 
Table 2 Comparison of Demographics and Fracture Characteristics Between the Two Groups

\begin{tabular}{|c|c|c|c|c|}
\hline Variables & Overall $(N=\mid 495)$ & Without POP $(\mathrm{N}=1442)$ & With POP $(\mathrm{N}=53)$ & $P$ value \\
\hline Age (>82, years), n (\%) & $5 I I(34.2)$ & $480(33.3)$ & $31(58.5)$ & $<0.001$ \\
\hline Sex (male), n (\%) & $547(36.6)$ & $483(33.5)$ & $28(52.8)$ & 0.004 \\
\hline Residential location(urban), n (\%) & $689(46.1)$ & $662(45.9)$ & $27(50.9)$ & 0.470 \\
\hline Hypertension, n (\%) & $724(48.8)$ & $716(49.7)$ & $8(45.3)$ & 0.532 \\
\hline Diabetes, n (\%) & $328(21.9)$ & $319(22.1)$ & $9(17.0)$ & 0.374 \\
\hline Cerebrovascular disease, n (\%) & $518(34.6)$ & $492(34.1)$ & $26(49.1)$ & 0.025 \\
\hline Cardiovascular disease, n (\%) & $60 I(40.2)$ & $565(39.2)$ & $36(67.9)$ & $<0.001$ \\
\hline Chronic respiratory disease, $\mathrm{n}(\%)$ & $8 I(5.4)$ & $69(4.8)$ & $12(22.6)$ & $<0.001$ \\
\hline Smoking history, n (\%) & $70(4.7)$ & $69(4.8)$ & I (I.9) & 0.327 \\
\hline Tumors, n (\%) & $36(2.4)$ & $36(2.5)$ & $0(0.0)$ & 0.244 \\
\hline Traumatic brain injury, $\mathrm{n}(\%)$ & $17(1.1)$ & $17(1.2)$ & $0(0.0)$ & 0.427 \\
\hline Liver disease, $\mathrm{n}(\%)$ & $33(2.2)$ & $28(1.9)$ & $5(9.4)$ & $<0.001$ \\
\hline Renal disease, n (\%) & $49(3.2)$ & $46(3.2)$ & $3(5.7)$ & 0.321 \\
\hline Urinary tract infection, $\mathrm{n}(\%)$ & $12(0.8)$ & $9(0.6)$ & $3(5.7)$ & $<0.001$ \\
\hline Rheumatoid diseases, n (\%) & $19(1.3)$ & $19(1.3)$ & $0(0.0)$ & 0.400 \\
\hline Previous surgical history & $235(15.7)$ & $227(15.7)$ & $8(15.1)$ & 0.899 \\
\hline Comorbidities(no.), n (\%) & & & & $<0.001$ \\
\hline 0 & $197(13.2)$ & $197(13.7)$ & $0(0.0)$ & \\
\hline I & $340(22.7)$ & $338(23.4)$ & $2(3.8)$ & \\
\hline$\geq 2$ & $958(64.1)$ & 907 (62.9) & $51(96.2)$ & \\
\hline ASA3-4, n (\%) & $802(53.6)$ & $765(53.1)$ & $37(69.8)$ & 0.016 \\
\hline BMI, n (\%) & & & & 0.011 \\
\hline$<18.5$ & $100(6.7)$ & $91(6.3)$ & $9(17.0)$ & \\
\hline $18.5-24.9$ & $860(57.5)$ & 831 (57.6) & $29(54.7)$ & \\
\hline $25-29.9$ & $483(32.3)$ & 47I (32.7) & $12(22.6)$ & \\
\hline$\geq 30$ & $52(3.5)$ & $49(3.4)$ & $3(5.7)$ & \\
\hline Injury mechanism (high energy), n (\%) & $80(5.4)$ & $79(5.5)$ & I (I.9) & 0.503 \\
\hline Side (left), n (\%) & $777(52.0)$ & $747(51.8)$ & $30(56.6)$ & 0.492 \\
\hline Transfer to ICU (yes), n (\%) & $43(1495)$ & $36(2.5)$ & $7(13.2)$ & $<0.001$ \\
\hline
\end{tabular}

Abbreviations: ASA, American Society of Anesthesiologists; BMI, body mass index; ICU, intensive care unit.

development of IHPOP is shown in Figure 2. Two of the 53 patients with IHPOP died while the others recovered.

The optimum cutoff value for quantitative data related to IHPOP was determined by ROC curve analysis (Table 1). The demographic and fracture characteristics are shown in Table 2. The average age was $78.9 \pm 7.5$ years; $34.2 \%$ of patients were male and $65.8 \%$ were female. The proportion of patients $>82$ years was higher in patients with IHPOP than in those without IHPOP $(58.5 \%$ vs $33.3 \%) \quad(p<0.001)$, whereas the opposite trend was observed for the proportion of female patients $(\mathrm{p}=0.004)$. The proportion of patients with $\geq 2$ comorbidities was $96.2 \%$ (51/53) in the IHPOP group and $62.9 \%$ in patients without IHPOP $(\mathrm{p}<0.001)$. More patients with IHPOP had cerebrovascular disease $(\mathrm{p}=0.025)$, cardiovascular disease $(p<0.001)$, chronic respiratory disease $(\mathrm{p}<0.001)$, liver disease $(\mathrm{p}<0.001)$, urinary tract infection $(\mathrm{p}<0.001)$, and an ASA score of 3-4 $(\mathrm{p}=0.016)$. There were also significant differences between the 2 groups in BMI $(p=0.011)$ and transfer to the intensive care unit (ICU; $\mathrm{p}<0.001)$. The percentage of patients treated with an intramedullary device was lower in the IHPOP group than in the non-IHPOP group, but the difference was not statistically significant $(\mathrm{p}=0.169$; Table 4$)$. Other characteristics were similar between the 2 groups.

Preoperative laboratory indices are shown in Table 3. Creatine kinase $(\mathrm{CK}) \mathrm{MB}>20 \mathrm{U} / \mathrm{l}(\mathrm{p}=0.027)$, B-type natriuretic peptide $(\mathrm{BNP})(\mathrm{p}<0.001)$, procalcitonin $(\mathrm{PCT}$; $\mathrm{p}=0.010)$, and $\mathrm{D}$-dimer $>2.26 \mathrm{mg} / \mathrm{l}(\mathrm{p}<0.001)$ were associated with an increased risk of developing IHPOP. The other variables showed no significant correlations, and no significant differences in surgical data were observed between the 2 groups (Table 4). However, the average 
Table 3 Comparison of Preoperative Laboratory Indicators Between the Two Groups

\begin{tabular}{|c|c|c|c|}
\hline Variables & Without POP $(\mathrm{N}=1945)$ & With POP $(\mathrm{N}=53)$ & $P$ value \\
\hline TP <65 g/L, n (\%) & $1185(82.2)$ & $47(88.7)$ & 0.222 \\
\hline ALB<35g/L, n (\%) & $975(67.6)$ & $37(69.8)$ & 0.737 \\
\hline GLOB (references $20-40 \mathrm{~g} / \mathrm{L}), \mathrm{n}(\%)$ & & & 0.693 \\
\hline$<20$ & $228(15.8)$ & $7(13.2)$ & \\
\hline$>40$ & $12(0.8)$ & $0(0.0)$ & \\
\hline A/G (references I.2-2.4), n (\%) & & & 0.676 \\
\hline$<1.2$ & $314(21.8)$ & $13(24.5)$ & \\
\hline$>2.4$ & $16(1.1)$ & $0(0.0)$ & \\
\hline ALT (references 9-50 U/L), n (\%) & & & 0.431 \\
\hline$<9$ & $162(11.2)$ & $8(15.1)$ & \\
\hline$>50$ & $72(5.0)$ & $\mathrm{I}(\mathrm{I} .9)$ & \\
\hline AST (references I5-40 U/L), n (\%) & & & 0.807 \\
\hline$<15$ & $269(18.7)$ & $9(17.0)$ & \\
\hline$>40$ & $140(9.7)$ & $4(7.5)$ & \\
\hline TBIL (>26), n (\%) & $192(13.3)$ & $9(17.0)$ & 0.442 \\
\hline DBIL (>6), n (\%) & $648(44.9)$ & $30(56.6)$ & 0.145 \\
\hline IBIL (>|4), n (\%) & $242(16.8)$ & $10(18.9)$ & 0.061 \\
\hline ALP (references 45-I25 U/L), n (\%) & & & 0.720 \\
\hline$<45$ & $169(11.7)$ & $7(13.2)$ & \\
\hline$>125$ & $92(6.4)$ & $2(3.8)$ & \\
\hline GGT (references I0-60 U/L), n (\%) & & & 0.936 \\
\hline$<10$ & $83(5.8)$ & $3(5.7)$ & \\
\hline$>60$ & $116(8.0)$ & $5(9.4)$ & \\
\hline CHE (references 5-12 U/L), n (\%) & & & 0.254 \\
\hline$<2$ & $627(43.5)$ & $29(54.7)$ & \\
\hline$>12$ & $5(0.3)$ & $0(0.0)$ & \\
\hline TBA (references I-I0 umol/L), n (\%) & & & 0.411 \\
\hline$<1$ & $91(6.3)$ & $3(5.7)$ & \\
\hline$>10$ & $164(11.4)$ & $3(5.7)$ & \\
\hline HCRP (>8mg/L), n (\%) & $1210(83.9)$ & $45(84.9)$ & 0.846 \\
\hline CK (>3IOU/L), n (\%) & $297(20.6)$ & $\mathrm{II}(20.8)$ & 0.978 \\
\hline CKMB (>20U/L), n (\%) & $219(15.2)$ & $14(26.4)$ & 0.027 \\
\hline LDH (>250U/L), n (\%) & $46 I(32.0)$ & $16(30.2)$ & 0.785 \\
\hline TC (>5.2 umol/L), n (\%) & $95(6.6)$ & $2(3.8)$ & 0.414 \\
\hline TG (>I.7 umol/L), n (\%) & $99(6.9)$ & $4(7.5)$ & 0.847 \\
\hline $\mathrm{Na}$ (references $137-147 \mathrm{mmol} / \mathrm{L}), \mathrm{n}(\%)$ & & & 0.471 \\
\hline$<137$ & $663(46.0)$ & $26(49.1)$ & \\
\hline$>147$ & $9(0.6)$ & $\mathrm{I}(1.9)$ & \\
\hline $\mathrm{K}$ (references $3.5-5.3 \mathrm{mmol} / \mathrm{L}), \mathrm{n}(\%)$ & & & 0.610 \\
\hline$<3.5$ & $205(14.2)$ & $6(11.3)$ & \\
\hline$>5.3$ & $16(1.1)$ & $0(0.0)$ & \\
\hline $\mathrm{CL}$ (references 99-II0 mmol/L), $\mathrm{n}(\%)$ & & & 0.050 \\
\hline$<99$ & $225(15.6)$ & $65(4.5)$ & \\
\hline$>110$ & $65(4.5)$ & $6(11.3)$ & \\
\hline
\end{tabular}


Table 3 (Continued).

\begin{tabular}{|c|c|c|c|}
\hline Variables & Without POP $(\mathrm{N}=1945)$ & With POP $(\mathrm{N}=53)$ & $P$ value \\
\hline TCO2(references $20-30 \mathrm{mmol} / \mathrm{L}), \mathrm{n}(\%)$ & & & 0.889 \\
\hline$<20$ & $69(4.8$ & $3(5.7)$ & \\
\hline$>30$ & $72 I(5.0)$ & $2(3.8)$ & \\
\hline GLU (>6.1), n (\%) & $868(60.2)$ & $34(64.2)$ & 0.761 \\
\hline UREA (>8), n (\%) & $327(22.8)$ & $15(28.3)$ & 0.320 \\
\hline CREA (references 57-97 mmol/L), n (\%) & & & 0.563 \\
\hline$<57$ & $623(43.2)$ & $20(37.7)$ & \\
\hline$>97$ & $92(6.4)$ & $5(9.4)$ & \\
\hline UA (references $208-428 \mathrm{mmol} / \mathrm{L}), \mathrm{n}(\%)$ & & & 0.302 \\
\hline$<208$ & $729(40.6)$ & $22(4 I .5)$ & \\
\hline$>428$ & $44(3.1)$ & $3(5.7)$ & \\
\hline CA (references $2.1 \mathrm{I}-2.52 \mathrm{mmol} / \mathrm{L}), \mathrm{n}(\%)$ & & & 0.600 \\
\hline$<2.11$ & $76 \mid(52.8)$ & $3 I(58.5)$ & \\
\hline$>2.52$ & $12(0.8)$ & $0(0.0)$ & \\
\hline $\mathrm{P}$ (references $0.85-1.5 \mathrm{I} \mathrm{mmol} / \mathrm{L}), \mathrm{n}(\%)$ & & & 0.579 \\
\hline$<0.85$ & $185(12.8)$ & $9(17.0)$ & \\
\hline$>1.51$ & $50(3.5)$ & $\mathrm{I}(1.9)$ & \\
\hline $\mathrm{Mg}$ (references $0.75-1.02 \mathrm{mmol} / \mathrm{L}), \mathrm{n}(\%)$ & & & 0.671 \\
\hline$<0.75$ & $167(11.6)$ & $44(3.1)$ & \\
\hline$>1.02$ & $8(15.1)$ & $\mathrm{I}(\mathrm{I} .9)$ & \\
\hline BNP (ng/L), n (\%) & & & $<0.001$ \\
\hline$<75$ & $558(38.7)$ & $9(17.0)$ & \\
\hline$>75$ & $416(28.8)$ & $30(56.6)$ & \\
\hline Unknown & $468(32.5)$ & $14(26.4)$ & \\
\hline WBC (references 3.5-9.5*10^9/L), n (\%) & & & 0.307 \\
\hline$<3.5$ & $10(0.7)$ & $0(0.0)$ & \\
\hline$>9.5$ & $513(35.6)$ & $24(45.3)$ & \\
\hline NEU (references 2.8-6.3*10^9/L), n (\%) & & & 0.071 \\
\hline$<1.8$ & $3(0.3)$ & $0(0.0)$ & \\
\hline$>6.3$ & $749(51.9)$ & $36(67.9)$ & \\
\hline LYM (references I.I-3.2*I0^9/L), n (\%) & & & 0.818 \\
\hline$<1.1$ & $752(52.1)$ & $29(54.7)$ & \\
\hline$>3.2$ & $8(0.6)$ & $0(0)$ & \\
\hline MON (references $\left.0.1-0.6^{*} 10^{\wedge} 9 / \mathrm{L}\right), \mathrm{n}(\%)$ & & & 0.520 \\
\hline$<0.1$ & $3(0.2)$ & $0(0)$ & \\
\hline$>0.6$ & $870(60.3)$ & $36(67.9)$ & \\
\hline EOS (references $\left.0.02-0.05 * 10^{\wedge} 9 / \mathrm{L}\right), \mathrm{n}(\%)$ & & & 0.052 \\
\hline$<0.02$ & $373(25.9)$ & $9(17.0)$ & \\
\hline$>0.52$ & $4(0.3)$ & $\mathrm{I}(\mathrm{I} .9)$ & \\
\hline BAS $(>0.06), n(\%)$ & $83(5.8)$ & $3(5.7)$ & 0.977 \\
\hline RBC (>5.8), n (\%) & $94(6.5)$ & $4(7.5)$ & 0.766 \\
\hline NEU\% (references 45-75\%), n (\%) & & & 0.650 \\
\hline$<45$ & $3(0.2)$ & $0(0.0)$ & \\
\hline
\end{tabular}

(Continued) 
Table 3 (Continued).

\begin{tabular}{|c|c|c|c|}
\hline Variables & Without POP $(\mathrm{N}=1945)$ & With POP $(\mathrm{N}=53)$ & $P$ value \\
\hline$>75$ & $865(60.0)$ & $35(66.0)$ & \\
\hline LYM\% (>5.2), n (\%) & $1378(95.6)$ & $50(94.3)$ & 0.673 \\
\hline MON\% (references 3-10\%), n (\%) & & & 0.638 \\
\hline$<3$ & $24(1.7)$ & $0(0.0)$ & \\
\hline$>10$ & $343(23.8)$ & $13(24.5)$ & \\
\hline BAS\% (>1\%), n (\%) & $10(0.7)$ & $\mathrm{II}(0.7)$ & 0.318 \\
\hline HGB (<1 I0/I20 g/L), n (\%) & 1334(92.5) & $50(94.3)$ & 0.618 \\
\hline MCV (references 82-100 fL), n (\%) & & & 0.086 \\
\hline$<82$ & $31(2.1)$ & $0(0.0)$ & \\
\hline$>100$ & $110(7.6)$ & $8(15.1)$ & \\
\hline MCH (references 27-34 pg), n (\%) & & & 0.189 \\
\hline$<27$ & $38(2.6)$ & $0(0.0)$ & \\
\hline$>34$ & $92(6.4)$ & $6(11.3)$ & \\
\hline MCHC (references 316-354 g/L), n (\%) & & & 0.074 \\
\hline$<316$ & $38(2.6)$ & $3(5.7)$ & \\
\hline$>354$ & $6 I(4.2)$ & $5(9.4)$ & \\
\hline RDW (references II.6-16.5\%), n (\%) & & & 0.912 \\
\hline$<11.6$ & $5(0.3)$ & $0(0.0)$ & \\
\hline$>16.5$ & $136(9.4)$ & $5(9.4)$ & \\
\hline PLT (references I25-350*10^9/L), n (\%) & & & 0.996 \\
\hline$<125$ & $135(9.4)$ & $5(9.4)$ & \\
\hline$>250$ & $86(6.0)$ & $3(5.7)$ & \\
\hline MPV (references7.4-II.0 fL), n (\%) & & & 0.246 \\
\hline$<7.4$ & $227(15.7)$ & $12(22.6)$ & \\
\hline$>11.0$ & $49(3.4)$ & $3(5.7)$ & \\
\hline РCT (references0.16-0.43\%), n (\%) & & & 0.010 \\
\hline$<0.16$ & $937(65.0)$ & $34(64.2)$ & \\
\hline$>0.43$ & $7(0.5)$ & $2(3.8)$ & \\
\hline PDW (references |2.0-18.1\%), n (\%) & & & 0.934 \\
\hline$<7.4$ & $130(9.0)$ & $4(7.5)$ & \\
\hline$>11.0$ & $26(1.8)$ & $\mathrm{I}(\mathrm{I} .9)$ & \\
\hline PT (> 2.5 S), n (\%) & $333(23.1)$ & $10(18.9)$ & 0.246 \\
\hline PTA (<80\%), n (\%) & $175(12.1)$ & $7(13.2)$ & 0.873 \\
\hline INR (>I.4\%), n (\%) & $\mathrm{II}(0.8)$ & $\mathrm{I}(\mathrm{I} .9)$ & 0.368 \\
\hline APTT (references28-42 S), n (\%) & & & 0.934 \\
\hline$<28$ & $500(34.7)$ & $24(45.3)$ & \\
\hline$>42$ & $20(1.4)$ & $0(0.0)$ & \\
\hline APTT-R (references0.7-I.3), n (\%) & & & 0.528 \\
\hline$<0.7$ & $18(1.2)$ & $0(0.0)$ & \\
\hline$>1.3$ & $16(1.1)$ & $0(0.0)$ & \\
\hline TT (references |4-2। S), n (\%) & & & 0.696 \\
\hline$<14$ & $1026(71.2)$ & $35(66.0)$ & \\
\hline
\end{tabular}

(Continued) 
Table 3 (Continued).

\begin{tabular}{|c|c|c|c|}
\hline Variables & Without POP $(\mathrm{N}=1945)$ & With POP $(\mathrm{N}=53)$ & $P$ value \\
\hline$>21$ & $15 I(10.5)$ & $6(11.3)$ & \\
\hline FIB (reference2.0-4.4 mg/L), $\mathrm{n}$ (\%) & & & 0.708 \\
\hline$<2.0$ & $16(1.1)$ & $0(0.0)$ & \\
\hline$>4.4$ & $350(24.3)$ & $14(26.4)$ & \\
\hline AT III (reference80-I20\%), n (\%) & & & 0.916 \\
\hline$<80$ & $295(20.5)$ & $12(22.6)$ & \\
\hline$>120$ & $33(2.3)$ & $\mathrm{I}(\mathrm{I} .9)$ & \\
\hline D-Dimer (>2.26 mg/L), n (\%) & $57 I(39.6)$ & $34(64.2)$ & $<0.001$ \\
\hline
\end{tabular}

Notes: RBC, red blood cell, reference range: female, 3.5-5.0*1012/L; males, 4.0-5.5*1012/L; HGB, hemoglobin, reference range: females, II0-I50g/L; males, I20-160g/L; HCT, haematocrit, 40-50\%; PLT, platelet, 100-300*109/L.

Abbreviations: ASA, American Society of Anesthesiologists; BMI, body mass index; TP, total protein; ALB, albumin; GLOB, globulin; A/G values, albumin/globulin; ALT, alanine transaminase; AST, aspartate aminotransferase; TBIL, total bilirubin; DBIL, direct bilirubin; IBIL, indirect bilirubin; ALP, alkaline phosphatase; GGT, $\gamma$-glutamyl transpeptidase; CHE, cholinesterase; TBA, total bile acid; HCRP, hypersensitive c-reactive protein; LDH, lactate dehydrogenase; CREA, creatinine; UA, uric acid; CA, calcium; P, phosphorus; Mg, magnesium; BNP, brain natriuretic peptide; WBC, white blood cell; NEUT, neutrophile; LYM, lymphocyte, MON, mononuclear cell; EOS, eosinophilic granulocyte; BAS, basophilic granulocyte; $\mathrm{MCV}$, mean corpuscular volume; $\mathrm{MCH}$, mean corpuscular hemoglobin; MCHC, mean corpuscular hemoglobin concentration; RDW, red blood cell distribution width; MPV, mean platelet volume; PCT, procalcitonin; pdw, platelet distribution width; PT, Prothrombin time; PTA, prothrombin activity; INR, international normalized ratio; APTT, activated partial thromboplastin time; APTT-R, activated partial thromboplastin time ratio; TT, thrombin time; TT-R, thrombin ratio; FIB, fibrinogen; AT III, antithrombin III.

Table 4 Comparison of Surgical Data Between the Two Groups

\begin{tabular}{|c|c|c|c|}
\hline Variables & Without HPPOP $(\mathrm{N}=\mid 442)$ & With HPPOP $(\mathrm{N}=53)$ & $P$ value \\
\hline Intraoperative blood loss (mL), n (\%) & $263.19(298.50)$ & $265.09(204.65)$ & 0.963 \\
\hline Intraoperative blood transfusion $(\mathrm{mL})$, mean(SD) & $133.67(318.19)$ & $105.19(229.72)$ & 0.519 \\
\hline Reduction methods (open reduction, $\mathrm{n}(\%)$ & $152(10.5)$ & $7(\mid 3.2)$ & 0.536 \\
\hline Surgeon (Deputy Chief Physician, n (\%) & $178(12.3)$ & $5(9.4)$ & 0.526 \\
\hline Time to surgery(days), mean (SD) & $5.19(3.93)$ & $6.15(4.92)$ & 0.083 \\
\hline Type of anesthesia (general, n (\%) & $59 I(4 I . I)$ & $18(34.0)$ & 0.307 \\
\hline Implant, n (\%) & & & 0.169 \\
\hline Intramedullary devices & |35| (93.7) & $53(100.0)$ & \\
\hline Extramedullary devices & $91(6.3)$ & $0(0.0)$ & \\
\hline Duration of surgery(mins), mean (SD) & $106.03(41.00)$ & $100.19(33.18)$ & 0.306 \\
\hline
\end{tabular}

time to surgery was $6.15 \pm 4.92$ days in the IHPOP group, which was longer than in the non-IHPOP group.

\section{Risk Factors for IHPOP}

Age $>82$ years, sex (male), cerebrovascular disease, cardiovascular disease, chronic respiratory disease, liver disease, urinary tract infection, ASA score of 3-4, number of comorbidities, transfer to ICU, BMI, PCT, BNP, D-dimer, and $\mathrm{CKMB}$ were included in the multivariate analysis (Table 5). Age $>82$ years $(\mathrm{OR}=2.54, \mathrm{p}=0.004)$, male sex $(\mathrm{OR}=2.13$, $\mathrm{p}=0.017)$, chronic respiratory disease $(\mathrm{OR}=5.02, \mathrm{p}<0.001)$, liver disease $(\mathrm{OR}=3.39, \mathrm{p}=0.037)$, urinary tract infection $(\mathrm{OR}=8.46, \mathrm{p}=0.005), \mathrm{CKMB}>20 \mathrm{U} / \mathrm{l}(\mathrm{OR}=2.31, \mathrm{p}=0.020)$, $\mathrm{BNP} \geq 75 \mathrm{ng} / \mathrm{l}(\mathrm{OR}=4.02 ; \mathrm{p}=0.001)$, and $\mathrm{D}-$ dimer $>2.26 \mathrm{mg} / \mathrm{l}$
$(\mathrm{OR}=2.69, \mathrm{p}=0.002)$ were identified as independent risk factors for the occurrence of IHPOP following geriatric intertrochanteric fracture surgery. The other factors were nonsignificant.

\section{Discussion}

The in-hospital death rate for hip fracture is $6-10 \% .^{22,23}$ IHPOP is a devastating complication for hip fracture patients - especially the geriatric population - that inevitably prolongs hospital stay and increases the risk of mortality. According to one report, $80 \%$ of patients with POP after hip fracture surgery are readmitted and $33.3 \%$ of these patients die within 1 year after surgery. ${ }^{13}$ The present study investigated the incidence of and risk factors for 
Table $5 \mathrm{OR}, 95 \% \mathrm{Cl}$, and $\mathrm{P}$ value for Independent Risk Factors in the Multivariable Logistic Regression Analysis of IHPOP

\begin{tabular}{|l|l|l|l|}
\hline Variables & OR & $\mathbf{9 5 \%} \mathbf{~ C l}$ & P value \\
\hline Age (>82 years) & 2.54 & $1.354-4.760$ & 0.004 \\
Gender(female) & 2.15 & $1.149-4.017$ & 0.017 \\
Chronic respiratory disease & 5.02 & $2.283-11.043$ & 0.000 \\
Liver disease & 3.39 & $1.074-10.675$ & 0.037 \\
Urinary tract infection & 8.46 & $1.922-37.193$ & 0.005 \\
CKMB(>20U/L) & 2.31 & $1.141-4.760$ & 0.020 \\
\hline BNP & & & 0.003 \\
$\quad \geq 75 \mathrm{ng} / \mathrm{L}$ & 4.02 & $1.795-9.010$ & 0.001 \\
Unknown & 2.98 & $1.199-7.384$ & 0.019 \\
\hline D-Dimer (>2.26 mg/L) & 2.69 & $1.440-5.006$ & 0.002 \\
\hline
\end{tabular}

Abbreviations: $\mathrm{OR}$, odds radio; $\mathrm{Cl}$, confidence interval; $\mathrm{CKMB}$, creatine phosphokinase isoenzyme; BNP, brain natriuretic peptide.

IHPOP following geriatric intertrochanteric fracture surgery at a trauma hospital in China. The incidence of IHPOP in 1495 consecutive patients was $3.5 \%$, and the multivariate analysis identified 8 independent risk factors associated with the occurrence of IHPOP including age, male sex, chronic respiratory disease, liver disease, urinary tract infection, CKMB, BNP, and D-dimer.

Advanced age is known to be correlated with the rate of POP after hip fracture. ${ }^{24,25}$ The average age of patients in our cohort was $78.9 \pm 7.5$ years, which is similar to previous studies. ${ }^{5}$ The optimum cutoff value for age related to IHPOP following geriatric intertrochanteric fracture surgery was 82 years by ROC curve analysis; age $>82$ years was an independent risk factor for the development IHPOP, and patients $>82$ years were 2.54 times more likely to develop IHPOP than those $\leq 82$ years. In agreement with our findings, it was previously reported that patients $>80$ years old were more susceptible to developing POP after hip fractures ${ }^{26}$ and that the incidence of POP increased with age, ${ }^{27}$ being $2.3,3.9$, and 5.6 times higher in patients aged $60-69,70-79, \geq 80$ years, respectively, than in those aged 50 years. Geriatric patients with intertrochanteric fractures are generally fragile and have at least one comorbidity. Moreover, the trauma of fracture and surgery can cause posttraumatic systemic inflammation and organ damage. ${ }^{28}$ Therefore, the number of comorbidities is a major contributor to the high risk of IHPOP following geriatric intertrochanteric fracture surgery.

Sex is a known risk factor for morbidity and mortality after hip fracture. ${ }^{18,29}$ In an analysis of 293 patients, male sex was an independent risk factor for POP in hip fracture patients over the age of 80 years $^{11}$ and in a retrospective cohort study, it was the strongest risk factor for POP after hip fracture. ${ }^{13}$ In our cohort, the ratio of males to females was $1: 1.7$, and male sex was an independent risk factor for the development of IHPOP following geriatric intertrochanteric fracture surgery. This result may be explained by the fact that more men

Table 6 All Parameters in This Study

\begin{tabular}{|l|l|}
\hline Clinical Data & Parameters \\
\hline $\begin{array}{l}\text { Demographics and fracture } \\
\text { characteristics }\end{array}$ & $\begin{array}{l}\text { Age, gender, residential location, hypertension, diabetes, cerebrovascular disease, cardiovascular disease, chronic } \\
\text { respiratory disease, smoking history, tumors, traumatic brain injury, liver disease, renal disease, urinary tract } \\
\text { infection, rheumatoid diseases, previous surgical history, the number of Comorbidities, ASA score, BMI, injury } \\
\text { mechanism, side and transfer to ICU. }\end{array}$ \\
\hline $\begin{array}{l}\text { Preoperative laboratory } \\
\text { indicators }\end{array}$ & $\begin{array}{l}\text { TP, ALB, GLOB, A/G values, ALT, AST, TBIL, DBIL, IBIL, ALP, GGT, CHE, TBA, HCRP, LDH, CK, CKMB, TC, Na, } \\
\text { MON\%, BAS\%, HGB, HCT, MCV, MCH, MCHC, RDW, PLT, MPV, PCT, PDW, PT, PTA, INR, APTT, APTT-R, TT, } \\
\text { TT-R, FIB, AT III and D-Dimer. }\end{array}$ \\
\hline Surgical data & $\begin{array}{l}\text { Intraoperative blood loss, intraoperative blood transfusion, reduction methods, surgeon, time to surgery, type of } \\
\text { anesthesia, implant (intramedullary or extramedullary devices), and duration of surgery }\end{array}$ \\
\hline
\end{tabular}

Abbreviations: ASA, American Society of Anesthesiologists score; BMI, body mass index; ICU, intensive care unit; TP, total protein; ALB, albumin; GLOB, globulin; A/G, albumin/globulin; ALT, alanine transaminase; AST, aspartate aminotransferase; TBIL, total bilirubin; DBIL, direct bilirubin; IBIL, indirect bilirubin; ALP, alkaline phosphatase; GGT, $\gamma$-glutamyl transpeptidase; CHE,cholinesterase; TBA, total bile acid; HCRP, hypersensitive c-reactive protein; LDH, lactate dehydrogenase; CK, creatine kinase; CKMB, creatine kinase myoglobin; TC, total cholesterol; Na, sodium; K, kalium; CL, chlorin; $\mathrm{TCO}_{2}$, Total carbon dioxide; GLU, glucose; CREA, urea, creatinine; UA, uric acid; CA, calcium; P, phosphorus; Mg, magnesium; BNP, brain natriuretic peptide; WBC, white blood cell; NEUT, neutrophile; LYM, lymphocyte; MON, mononuclear cell; EOS, eosinophilic granulocyte; BAS, basophilic granulocyte; RBC, red blood cell; HGB, hemoglobin; HCT, haematocrit; MCV, mean corpuscular volume; MCH, mean corpuscular hemoglobin; MCHC, mean corpuscular hemoglobin concentration; RDW, red blood cell distribution width; PLT, platelet; MPV, mean platelet volume; PCT, procalcitonin; PDW, platelet distribution width; PT, prothrombin time; PTA, prothrombin activity; INR, international normalized ratio; APTT, activated partial thromboplastin time; APTT-R, activated partial thromboplastin time ratio; TT, thrombin time; TT-R, thrombin ratio; FIB, fibrinogen; AT III, antithrombin III. 
are smokers and therefore have a worse respiratory condition than women. Indeed, we found that chronic respiratory disease was significantly associated with increased risk of IHPOP following geriatric intertrochanteric fracture surgery. Moreover, liver disease $(\mathrm{OR}=3.39$, $\mathrm{p}=0.037)$ and urinary tract infection $(\mathrm{OR}=8.46, \mathrm{p}=0.005)$ were also correlated with an elevated risk of IHPOP, which has not been previously reported.

Some preoperative laboratory indices including CKMB $>20 \mathrm{U} / \mathrm{l}(\mathrm{OR}=2.31, \mathrm{p}=0.020), \mathrm{BNP} \geq 75 \mathrm{ng} / \mathrm{l}(\mathrm{OR}=4.02$, $\mathrm{p}=0.001)$, and $\mathrm{D}$-dimer $>2.26 \mathrm{mg} / \mathrm{l}(\mathrm{OR}=2.69, \mathrm{p}=0.002)$ were independent risk factors for IHPOP following geriatric intertrochanteric fracture surgery. BNP is a biomarker that has been used extensively in perioperative treatment of orthopedic surgery patients. ${ }^{30,31}$ However, there have been no studies investigating the relationship between BNP and POP after intertrochanteric fracture. We determined that a BNP cutoff value $>75 \mathrm{ng} / \mathrm{l}(\mathrm{OR}=4.02, \mathrm{p}=0.001)$ was associated with increased risk of IHPOP following geriatric intertrochanteric fracture surgery. Villacorta Junior et $\mathrm{al}^{32}$ found that BNP $>60 \mathrm{ng} / \mathrm{l}$ was also an independent risk factor for postoperative adverse cardiac events after orthopedic surgery. This association between BNP and adverse cardiac events could explain our observation. Although D-dimer has prognostic value for deep vein thrombosis, it is unclear whether it is useful for predicting the development of POP. Our results showed that D-dimer $>2.26 \mathrm{mg} / 1$ increased the risk for the development of IHPOP; however, further studies are needed to clarify the physiologic link between BNP or D-dimer and POP in geriatric patients who have undergone intertrochanteric fracture surgery.

We acknowledge some limitations to our study. It involved only one institution, and some diseases such as dementia and Parkinson's disease that may have contributed to an increased incidence of IHPOP were not included in our analysis. Multicenter studies should be conducted to investigate the incidence of and risk factors for IHPOP within 30 days or 1 year of intertrochanteric fracture surgery to confirm our findings.

Nonetheless, this is the largest and most comprehensive investigation of IHPOP incidence and risk in geriatric patients who specifically underwent intertrochanteric fracture surgery. A total of 1495 elderly patients ( $>65$ years) and $>90$ variables that could influence IHPOP incidence were analyzed (Table 6). Additionally, all patient data were extracted from a prospective database, which eliminated selection and recall bias; and quantitative data were evaluated by ROC curve analysis to identify the most sensitive cutoff value.

\section{Conclusion}

In conclusion, we found that the incidence of IHPOP following geriatric intertrochanteric fracture surgery was 3.5\%. Age, male sex, chronic respiratory disease, liver disease, urinary tract infection, $\mathrm{CKMB}, \mathrm{BNP}$, and D-dimer were significant risk factors for the occurrence of IHPOP after the surgery, and the implant (intramedullary devices or extramedullary device) was not independent risk factor for the developing of IHPOP. Targeted preoperative management based on risk factors can potentially reduce the risk of IHPOP in geriatric patients undergoing surgery for intertrochanteric fractures and thereby improve their chances of full recovery.

\section{Acknowledgments}

We thank RP Zhang of the Department of Orthopedics and YC Yin of the Department of Statistics and Applications for their generous assistance.

\section{Disclosure}

All authors declare that they have no conflicts of interest for this work.

\section{References}

1. Zhang Y. Clinical epidemiology of orthopaedic trauma. Thieme. 2012.

2. Klop C, Welsing PM, Cooper C, et al. Mortality in British hip fracture patients, 2000-2010: a population-based retrospective cohort study. Bone. 2014;66:171-177. doi:10.1016/j.bone.2014.06.011

3. Schmidt AH, Braman JP, Duwelius PJ, et al. Geriatric trauma: the role of immediate arthroplasty. J Bone Joint Surg Am. 2013;95 (24):2230-2239.

4. Socci AR, Casemyr NE, Leslie MP, et al. Implant options for the treatment of intertrochanteric fractures of the hip: rationale, evidence, and recommendations. Bone Joint J. 2017;99-B(1):128-133. doi:10.1302/0301-620X.99B1.BJJ-2016-0134.R1

5. Mattisson L, Bojan A, Enocson A. Epidemiology, treatment and mortality of trochanteric and subtrochanteric hip fractures: data from the Swedish fracture register. BMC Musculoskelet Disord. 2018;19(1):369. doi:10.1186/s12891-018-2276-3

6. Pedersen AB, Christiansen CF, Gammelager H, et al. Risk of acute renal failure and mortality after surgery for a fracture of the hip: a population-based cohort study. Bone Joint J. 2016;98-B (8):1112-1118. doi:10.1302/0301-620X.98B8.37497

7. Chatterton BD, Moores TS, Ahmad S, et al. Cause of death and factors associated with early in-hospital mortality after hip fracture. Bone Joint J. 2015;97-B(2):246-251. doi:10.1302/0301-620X.97B2.35248

8. Roche JJ, Wenn RT, Sahota O, et al. Effect of comorbidities and postoperative complications on mortality after hip fracture in elderly people: prospective observational cohort study. BMJ. 2005;331 (7529):1374. doi:10.1136/bmj.38643.663843.55 
9. Panula J, Pihlajamäki H, Mattila VM, et al. Mortality and cause of death in hip fracture patients aged 65 or older: a population-based study. BMC Musculoskelet Disord. 2011;12:105. doi:10.1186/14712474-12-105

10. Lv H, Yin P, Long A, et al. Clinical characteristics and risk factors of postoperative pneumonia after hip fracture surgery: a prospective cohort study. Osteoporos Int. 2016;27(10):3001-3009. doi:10.1007/ s00198-016-3624-5

11. Wang X, Dai L, Zhang Y, et al. Gender and low albumin and oxygen levels are risk factors for perioperative pneumonia in geriatric hip fracture patients. Clin Interv Aging. 2020;15:419-424. doi:10.2147/ CIA.S241592

12. Kjørholt KE, Kristensen NR, Prieto-Alhambra D, et al. Increased risk of mortality after postoperative infection in hip fracture patients. Bone. 2019;127:563-570. doi:10.1016/j.bone.2019.07.023

13. Bohl DD, Sershon RA, Saltzman BM, et al. Incidence, risk factors, and clinical implications of pneumonia after surgery for geriatric hip fracture. J Arthroplasty. 2018;33(5):1552-1556.e1. doi:10.1016/j. arth.2017.11.068

14. Parker M, Johansen A. Hip fracture. BMJ. 2006;333(7557):27-30. doi:10.1136/bmj.333.7557.27

15. Shin WC, Do MU, Woo SH, et al. C-reactive protein for early detection of postoperative systemic infections in intertrochanteric femoral fractures. Injury. 2018;49(10):1859-1864. doi:10.1016/j. injury.2018.07.029

16. Heckmann N, Hill JR, Vakhshori V, et al. Increased pulmonary complications associated with intramedullary fixation of intertrochanteric fractures: an analysis of 13,276 hips. J Am Acad Orthop Surg. 2019;27(18):690-695. doi:10.5435/JAAOS-D-17-00921

17. Wang Y, Li X, Ji Y, et al. Preoperative serum albumin level as a predictor of postoperative pneumonia after femoral neck fracture surgery in a geriatric population. Clin Interv Aging. 2019;14:2007-2016. doi:10.2147/CIA.S231736

18. Sheehan KJ, Guerrero EM, Tainter D, et al. Prognostic factors of in-hospital complications after hip fracture surgery: a scoping review. Osteoporos Int. 2019;30(7):1339-1351. doi:10.1007/s00198-01904976-x

19. Kempegowda H, Richard R, Borade A, et al. Obesity is associated with high perioperative complications among surgically treated intertrochanteric fracture of the femur. J Orthop Trauma. 2017;31 (7):352-357. doi:10.1097/BOT.0000000000000825

20. Fields AC, Dieterich JD, Buterbaugh K, et al. Short-term complications in hip fracture surgery using spinal versus general anaesthesia. Injury. 2015;46(4):719-723. doi:10.1016/j.injury.2015.02.002
21. Bohl DD, Shen MR, Hannon CP, et al. Serum albumin predicts survival and postoperative course following surgery for geriatric hip fracture. J Bone Joint Surg Am. 2017;99(24):2110-2118. doi:10.2106/JBJS.16.01620

22. Bohm E, Loucks L, Wittmeier K, et al. Reduced time to surgery improves mortality and length of stay following hip fracture: results from an intervention study in a Canadian health authority. Can J Surg. 2015;58(4):257-263. doi:10.1503/cjs.017714

23. Sheehan KJ, Sobolev B, Guy P, et al. In-hospital mortality after hip fracture by treatment setting. CMAJ. 2016;188(17-18):1219-1225. doi:10.1503/cmaj.160522

24. Jain S, Self WH, Wunderink RG, et al. Community-acquired pneumonia requiring hospitalization among U.S. adults. $N$ Engl J Med. 2015;373(5):415-427. doi:10.1056/NEJMoa1500245

25. Flikweert ER, Wendt KW, Diercks RL, et al. Complications after hip fracture surgery: are they preventable? Eur J Trauma Emerg Surg. 2018;44(4):573-580. doi:10.1007/s00068-017-0826-2

26. Chang SC, Lai JI, Lu MC, et al. Reduction in the incidence of pneumonia in elderly patients after hip fracture surgery: an inpatient pulmonary rehabilitation program. Medicine. 2018;97(33):e11845. doi:10.1097/MD.0000000000011845

27. Smetana GW, Lawrence VA, Cornell JE, American College of Physicians; Preoperative pulmonary risk stratification for noncardiothoracic surgery: systematic review for the American College of Physicians. Ann Intern Med. 2006;144(8):581-595. doi:10.7326/ 0003-4819-144-8-200604180-00009

28. Yu X, Chen X, Sun T. MicroRNA-205-5p targets HMGB1 to suppress inflammatory responses during lung injury after hip fracture. Biomed Res Int. 2019;2019:7304895. doi:10.1155/2019/7304895

29. Bennet SJ, Berry OM, Goddard J, et al. Acute renal dysfunction following hip fracture. Injury. 2010;41(4):335-338. doi:10.1016/j. injury.2009.07.009

30. Pili-Floury S, Ginet M, Saunier L, et al. Preoperative plasma B-type natriuretic peptide (BNP) identifies abnormal transthoracic echocardiography in elderly patients with traumatic hip fracture. Injury. 2012;43(6):811-816. doi:10.1016/j.injury.2011.09.016

31. Park JH, Shin GJ, Ryu JI, et al. Postoperative B-type natriuretic Peptide levels associated with prolonged hospitalization in hypertensive patients after non-cardiac surgery. Korean Circ J. 2012;42 (8):521-527. doi:10.4070/kcj.2012.42.8.521

32. Villacorta Junior H, Castro IS, Godinho M, et al. B-type natriuretic peptide is predictive of postoperative events in orthopedic surgery. Arq Bras Cardiol. 2010;95(6):743-748. doi:10.1590/s0066$782 \times 2010005000131$
Clinical Interventions in Aging

\section{Publish your work in this journal}

Clinical Interventions in Aging is an international, peer-reviewed journal focusing on evidence-based reports on the value or lack thereof of treatments intended to prevent or delay the onset of maladaptive correlates of aging in human beings. This journal is indexed on PubMed Central, MedLine, CAS, Scopus and the Elsevier
Bibliographic databases. The manuscript management system is completely online and includes a very quick and fair peer-review system, which is all easy to use. Visit http://www.dovepress.com/ testimonials.php to read real quotes from published authors. 